\title{
Increased m6A methylation level is associated with the progression of human abdominal aortic aneurysm
}

\author{
Yuchen $\mathrm{He}^{1}$, Jia Xing ${ }^{2}$, Shiyue Wang ${ }^{1}$, Shijie Xin ${ }^{1}$, Yanshuo Han ${ }^{3,4}$, Jian Zhang ${ }^{1}$ \\ ${ }^{1}$ Department of Vascular Surgery, The First Hospital of China Medical University, Key Laboratory of Pathogenesis, Prevention, and Therapeutics \\ of Aortic Aneurysm, Shenyang 110001, China; ${ }^{2}$ Department of Histology and Embryology, China Medical University, Shenyang 110122, China; \\ ${ }^{3}$ Department of General Surgery, Shengjing Hospital of China Medical University, Shenyang 110004, China; ${ }^{4}$ School of Life Science and Medicine, \\ Dalian University of Technology (DUT), Panjin 124221, China \\ Contributions: (I) Conception and design: Y He, Y Han, J Zhang; (II) Administrative support: J Zhang, S Xin; (III) Provision of study materials or \\ patients: Y He, J Xing; (IV) Collection and assembly of data: Y He, S Wang; (V) Data analysis and interpretation: Y He; (VI) Manuscript writing: All \\ authors; (VII) Final approval of manuscript: All authors. \\ Correspondence to: Yanshuo Han, MD. Department of General Surgery, Shengjing Hospital of China Medical University, Shenyang 110004, China; \\ School of Life Science and Medicine, Dalian University of Technology (DUT), Panjin 124221, China. Email: yanshuohan@cmu.edu.cn; Jian Zhang, \\ MD, PhD. Department of Vascular Surgery, The First Hospital of China Medical University, Key Laboratory of Pathogenesis, Prevention, and \\ Therapeutics of Aortic Aneurysm, Shenyang 110001, China. Email: jianzhang@cmu.edu.cn.
}

Background: The role of N6-methyladenosine (m6A) modification in abdominal aortic aneurysm (AAA) has not been extensively studied. This study therefore aimed to investigate m6A RNA methylation and the expressions of the corresponding modulators in AAA.

Methods: A comparative study between AAA tissue samples ( $\mathrm{n}=32)$ and healthy aortas $(\mathrm{n}=12)$ was performed using m6A methylation quantification for messenger RNA (mRNA) m6A status, quantitative polymerase chain reaction (qPCR), and western blot for the expressions of m6A modulators and immunohistochemistry (IHC) to detect locations of the modulators in AAA tissues.

Results: The m6A level significantly increased in AAA as compared to healthy aorta tissues. Among AAA patients, the high m6A level represented an even greater risk of AAA rupture as compared to nonruptured AAA [odds ratio (OR), 1.370; 95\% confidence interval (CI), 1.007-1.870]. The major N6adenosine modulators, including YTHDF1, YTHDF3, FTO, and METTL14, are the main factors involved in aberrant m6A modification and the expression of both was significantly correlated to the proportion of m6A in total mRNA. Clinically, YTHDF3 represented an even greater risk of rupture (OR, 1.036; 95\% CI, 1.001-1.072). Regarding the cellular location, METTL14 seemed to be associated with inflammatory infiltrates and neovascularization. Furthermore, a strong correlation was seen between FTO and aneurysmal smooth muscle cells (SMCs), Y'THDF3, and macrophage infiltrate.

Conclusions: We were first to observe m6A modification in human AAA tissues. The results also reveal the important roles of m6A modulators, including YTHDF3, FTO, and METTL14, in the pathogenesis of human AAA and provide a new view on m6A modification in AAA. Our findings suggest a potential mechanism of epigenetic alterations in clinical AAA.

Keywords: Abdominal aortic aneurysm (AAA); N6-methyladenosine modification (m6A modification); N6methyladenosine RNA methyltransferase (m6A RNA methyltransferase); epigenetics

Submitted Aug 26, 2019. Accepted for publication Nov 29, 2019.

doi: $10.21037 /$ atm.2019.12.65

View this article at: http://dx.doi.org/10.21037/atm.2019.12.65 


\section{Introduction}

For the elderly, abdominal aortic aneurysm (AAA) is a common vascular condition. The overall mortality rate for people over the age of 65 from AAA in developed countries is $1.3 \%$ (1). Surgical repair is currently the only systematic therapeutic solution, including for a dilated aorta or ruptured AAA (2-4) open surgical repair (OSR) or endovascular repair (EVAR). As is commonly accepted, inflammatory cell infiltration is one of its characteristics $(5,6)$. In addition, smooth muscle cells (SMCs) apoptosis is involved in extracellular matrix (ECM) degradation in AAA (6), while angiogenesis is an integral process of AAA (7).

Nevertheless, the exact cellular mechanisms contributing to AAA in each patient has yet to be elucidated. Therefore, a better understanding of these cellular mechanisms and regulatory networks driving AAA development and progression are essential in identifying novel therapeutic targets.

The importance of epigenetic alterations, including DNA methylation, histone modification, and RNA modification, through their robust effects on gene expression, are being increasingly recognized for many cardiovascular diseases. Our previous studies have reported that aberrant epigenetic modifications, such as changes in histone acetylation, are present in AAA (8). These findings may offer new insight into the pathologic mechanisms of AAA. N6-methyladenosine (m6A) is a methylated adenosine base at the nitrogen- 6 position. This post-transcriptional modification is very common in the eukaryotic messenger RNA (mRNA) (9-12). m6A was first identified in mRNAs from Novikoff hepatoma cells (13). The potential functions of m6A mRNA modification in cancer $(9,14-16)$ and other diseases $(17,18)$ have already been reported. m6A is highly enriched in the 3'-UTRs of certain mRNAs and selectively regulates the stability of target mRNAs and thus indirectly affects gene expression $(13,19,20)$.

$\mathrm{m} 6 \mathrm{~A}$ modification is regulated by several modulators that are characterized as "writers", "erasers", and "readers" according to their functions $(13,21)$. The m6A writers, including METTL3, METTL14, and WTAP, constitute the m6A methylase complex, which activates m6A modification (9,18,22-24). The m6A erasers, including ALKBH5 and FTO, act as demethylases to reverse the m6A modification $(15,25)$. Finally, m6A readers recognize m6Amodified mRNAs and transmit the code to downstream effectors. The members of the YTH domain-containing family, including YTHDF1, YTHDF2, and YTHDF3, are vital m6A-binding proteins. To our knowledge, m6Amodified mRNAs have not been addressed experimentally in AAA.

Therefore, we focused on m6A mRNA modification in human AAA tissue samples, including the evaluation of the extent of m6A mRNA methylation and expression of the corresponding modulators of the m6A modification. Additionally, we analyzed the correlation between m6A modification and patient clinical data. Our results show there to be an important function of the m6A modification in AAA progression.

\section{Methods}

\section{China Medical University aneurysm biobank and tissue collection}

Between January 2009 and December 2018, human AAA tissues, corresponding peripheral blood samples, and clinical data were collected from 126 consecutive patients in the Department of Vascular Surgery of The First Hospital of China Medical University (CMU) and the Department of General Surgery of Shengjing Hospital of CMU immediately after open aneurysmal surgery as described previously (26). Written informed consent was obtained from all patients. Human AAA samples collection conducted according to the Guidelines of the World Medical Association Declaration of Helsinki, and was approved by the Ethics Committees of Shenging Hospital of China Medical University (ethical approval number: 2016PS085K).

Aneurysm tissues were obtained from 126 Chinese patients with AAA during elective OSR. Initially, an experienced vascular surgeon examined all patients within 2 days before and after the operation. The indication for surgical treatment was based on international guidelines (27). In most cases, computed tomography angiography (CTA) was performed to achieve detailed imaging of the AAA. Inclusion criteria included the following: (I) patients with an infrarenal abdominal aorta diameter above $30 \mathrm{~mm}$ or larger than 1.5-2 times the diameter of the abdominal aorta in their normal segment diagnosed by CTA; and (II) availability of corresponding patient history and clinical data, including history of rupture, history of coronary and peripheral artery disease, medication history, and the presence of risk factors for atherosclerosis, such as smoking habits, hypertension, diabetes mellitus (DM) or hyperlipidemia. Exclusion criteria for the AAA group 
included Ehlers-Danlos syndrome, Marfan syndrome, and other known vascular or connective tissue disorders.

Maximal diameters of AAAs were measured during the surgery, and the AAA specimen were collected from anterior part of abdominal aortas, where dilatation was judged to be maximal. The aortic tissue was sufficient quality of aneurysm tissue for histological and morphological characterization. As of December 2018, 9 patients did not willingly or openly provide their inpatient information for publication, including their clinical data, aneurysmal tissue, or blood samples. Finally, aneurysm specimens from 117 patients were eligible for further analyses. Patients registered in the CMU Aneurysm Biobank (CMUaB) were selected for this study to identify patients with a recognized AAA. Peripheral blood mononuclear cells (PBMCs) of partial 117 patients were extracted by Ficoll-sodium diatrizoate density gradient centrifugation, as described previously (26). Moreover, AAA tissue samples were collected using standard procedures from the anterior sac of the infrarenal abdominal aorta and divided into two parts: one was stored in liquid nitrogen, and the other was fixed in 4\% paraformaldehyde for paraffin sectioning. The selection process of the included patients is summarized in Figure 1.

For the current study, AAA tissues from 32 patients (30 males and 2 females) with an AAA were available from the tissue collection as were the healthy aorta tissues of a group of 12 matched controls. Control tissue samples were obtained from organ donors. Exclusion criteria for the control group included cancer, drug history, infection, or any other immune-related disease that might have influenced the study.

\section{$R N A$ extraction and total mRNA m6A level determination}

Total mRNA was extracted from aortic tissue samples using TRIzol reagent (TaKaRa Bio, Shiga, Japan), as described previously (8). An m6A RNA methylation quantification kit (Epigentek) was purchased for directly detecting m6A RNA methylation status using total mRNA. The percentage of m6A modified mRNA in the total mRNA (m6A\%) was used to evaluated mRNA m6A methylation status in this study. In brief, according to the manufacturer's protocol, $200 \mathrm{ng}$ mRNA from each sample was allocated by pipette into assay wells. Subsequently, a capture antibody specific for m6A was added to the wells. After washing, a detection antibody was added to the wells. The developer solution was added to each well following the wash steps to remove any liquid while protecting samples from light. The color development was arrested, and the intensity of color was measured at $450 \mathrm{~nm}$ wavelength. The proportion of m6A in total mRNA was calculated as a percentage according to the standard curve.

\section{A quantitative real-time polymerase chain reaction ( $q R T$ - PCR) analysis}

qRT-PCR was performed using Universal SYBR qPCR Master Mix (Q711-02/03, Vazyme Bio) after the synthesis of cDNA using RT SuperMix kits (R223-01; Vazyme Bio) with a modified amplification protocol: initial denaturation step at $95^{\circ} \mathrm{C}$ for $30 \mathrm{~s}$, then 40 cycles of $95^{\circ} \mathrm{C}$ denaturation for $5 \mathrm{~s}$, and annealing and extension at $60{ }^{\circ} \mathrm{C}$ for $30 \mathrm{~s}$ each. RT-PCR analysis for all samples was independently repeated at least twice. Normalization was performed with GAPDH. The RNA specific primers were listed in Table 1.

\section{Western blotting analysis}

For RNA methyltransferases, demethylases, and m6Abinding proteins with significant differences between AAA and control groups at the mRNA level, the samples were further subjected to western blotting analysis. Proteins were extracted from fresh frozen samples using RIPAbased reagents according to a standard protocol and then separated by sodium dodecyl sulfate-polyacrylamide gel electrophoresis. The separated proteins were then transferred onto polyvinylidene difluoride membranes. After blocking with non-fat milk and incubation with appropriate primary and secondary antibodies, the membranes were then developed with an ECL detection system according to the manufacturer's protocol. Normalization was performed with GAPDH. The primary antibodies used in this study were anti-YTHDF3 (ab103328; Abcam, Cambridge, UK; dilution 1:1,000), anti-YTHDF1 (Cat. No. 17479-AP; Proteintech, Wuhan, China; dilution 1:1,000), anti-FTO (ab124892; Abcam; dilution 1:1,000), anti-METTL14 (abs128743; Absin, Shanghai, China dilution 1:1,000), and anti-GAPDH (dilution 1:2,000; Zsbio, Beijing, China).

\section{Histology and immunobistochemistry (IHC)}

Representative sections of aortic tissue samples $(2-3 \mu \mathrm{m})$ were used for histological and immunohistochemical analyses. Paraffin sections were routinely stained with hematoxylin-eosin (HE) to assess tissue morphology, cellular composition, and degree of infiltration with inflammatory cells in all AAA samples as described previously (8). The 

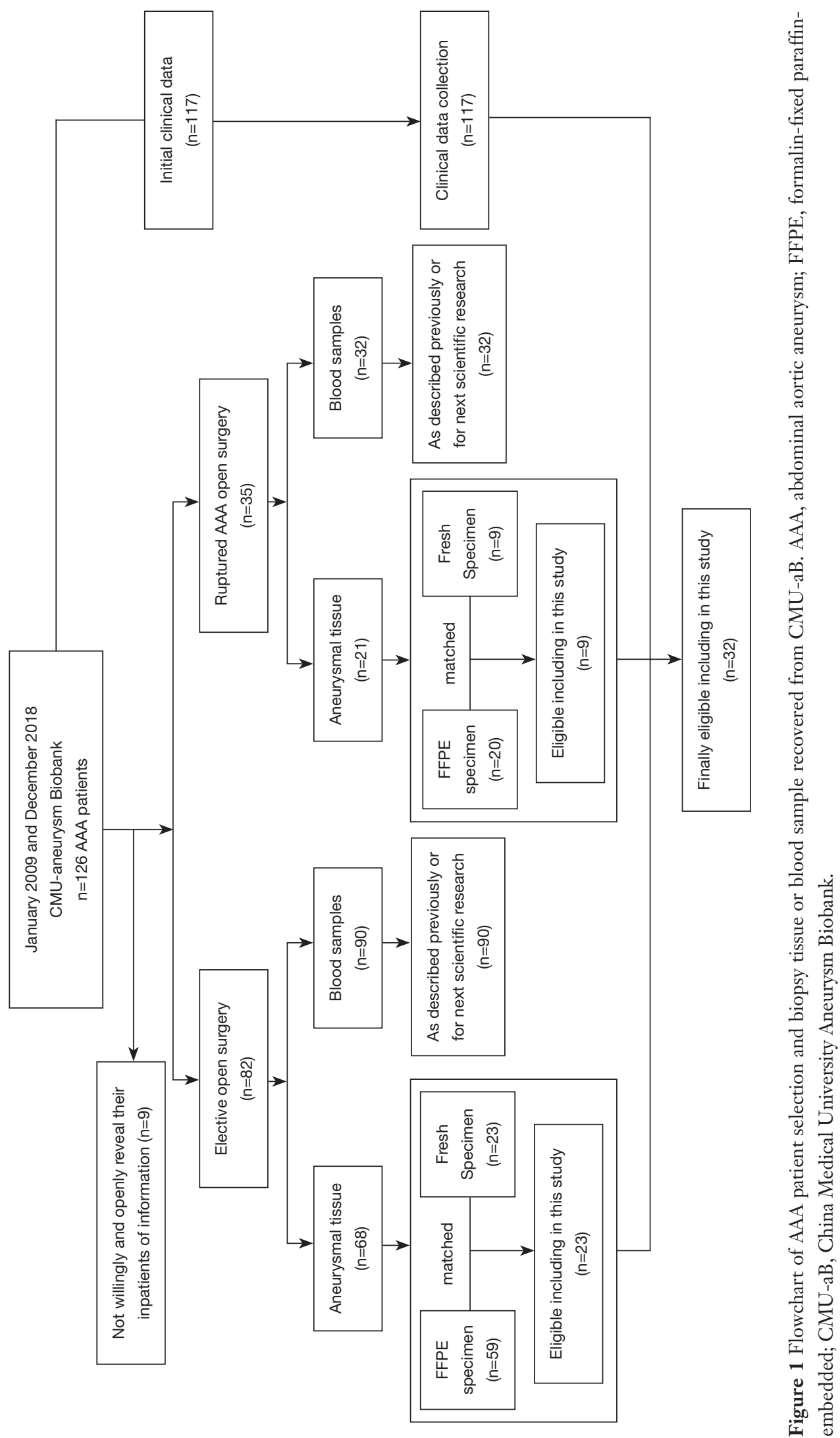


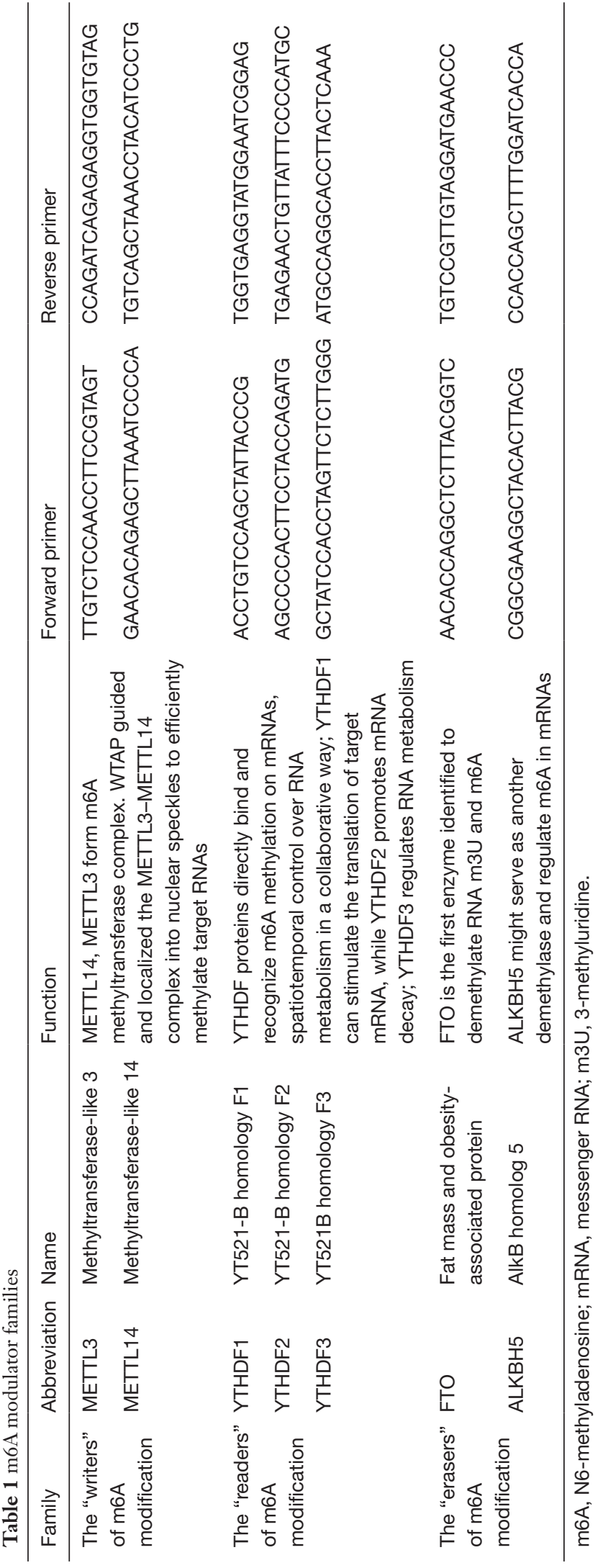

histological evaluation process is detailed below. The quality of each slide was assessed by two independent investigators as follows: no staining (-), weak positive staining $(+/-)$, scattered positive staining $(+)$, majority positive staining $(++)$, and strong overall positive staining $(+++)$. These score analyses were performed for all cells within the vessel wall and all specimens. This histopathological grading was performed for endothelial cells (ECs), inflammatory infiltrate, and macrophages in the AAA tissue samples and healthy aortas.

For IHC, tissue sections were boiled to retrieve antigen epitopes, washed, and treated with appropriate antibodies followed by dewaxing and hydration. For the analysis of cells in the AAA wall, smooth muscle cells were detected with anti- $\alpha$-SMA (dilution 1:400; Absin, Shanghai, China), ECs with anti-CD34 (dilution 1:100; Proteintech, Wuhan, China), monocytes (MONOs) with anti-CD68 (dilution 1:800; Absin), leukocytes with antiCD45 (dilution 1:500; Proteintech), and T-lymphocytes with anti-CD3 (dilution 1:800; Absin). Anti-FTO, antiYTHDF3, and METTL14 were applied for the detection of m6A modulators in AAA samples according to the manufacturer's instructions.

\section{Analysis of histology and IHC}

All stained tissue samples (slides) were captured with the digital image capture device Nikon ScanScope 90i system (Nikon, Japan). More precisely, a five-slide load capacity and $20 \times$ and $40 \times$ magnification of the $90 \mathrm{i}$ system were used in this study as digitalization image capture settings.

For conventional and standard staining, the histological classification was performed by characterizing vessel wall cellularity and degree of calcification. Subsequently, the grade was assessed by the baseline cell count and intensity of corresponding cells, including SMCs, infiltrates macrophages, and neovascularization.

Consecutive slides from each specimen were prepared and incubated with the appropriate antibodies to determine the expression of the biomarker in the individual cells within the aneurysmal lesions. In all cases, one slide was stained with an antibody to detect the specific cell type, and a consecutive slide was stained with the antibody against the individual biomarker of interest.

\section{Statistical analysis}

SPSS 22.0 (SPSS Inc., Chicago, IL, USA) was used 
Table 2 Demographic and clinical characteristics of AAA patients and controls included in this study

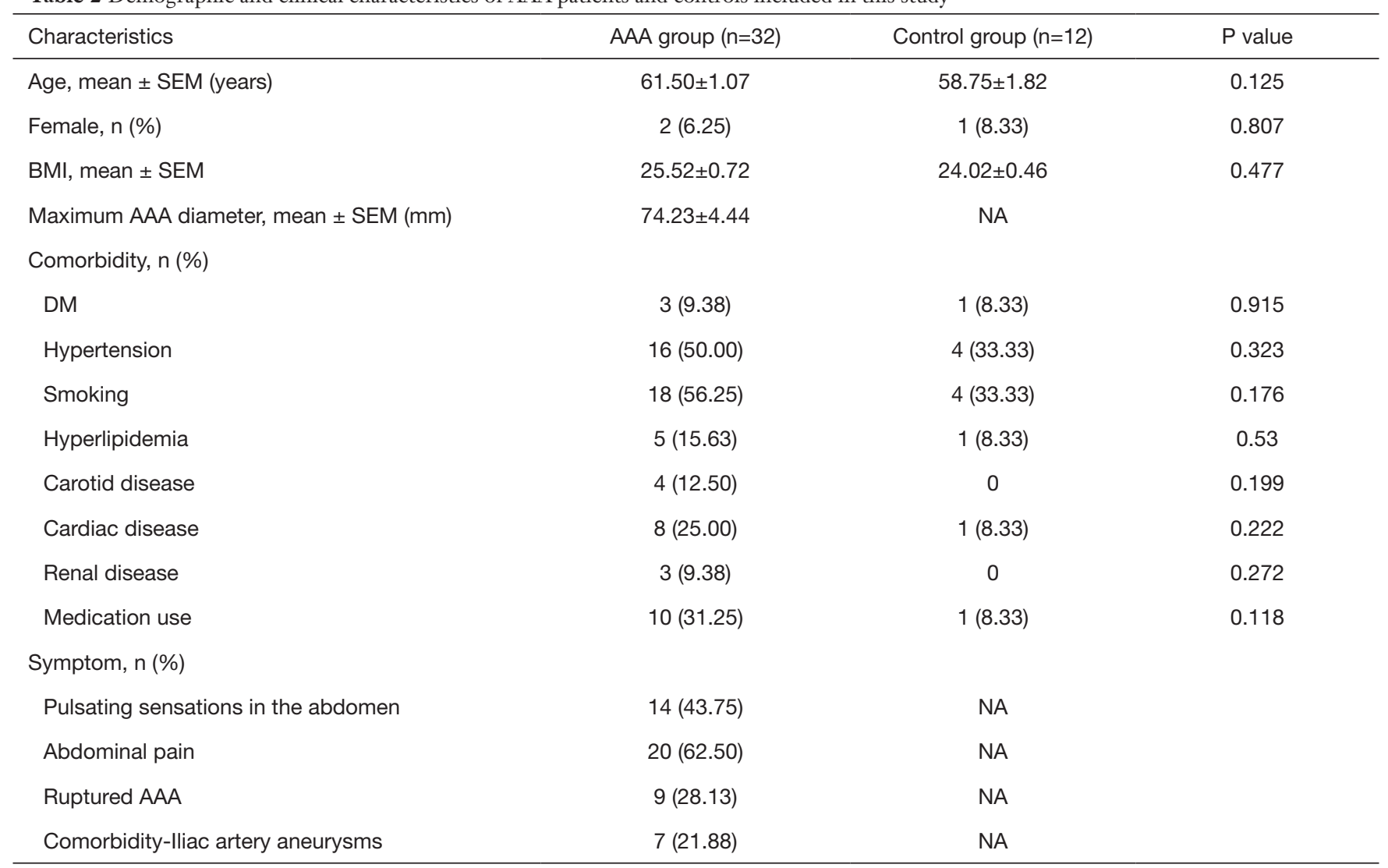

AAA, abdominal aortic aneurysm; SEM, standard error of mean; BMI, body mass index; DM, diabetes mellitus; NA, not available.

for statistical analysis. Chi-square was used to examine differences in categorical variables between the groups. We examined data distribution with the one-sample KolmogorovSmirnov test. Either the parametric $t$-test for unpaired samples or the non-parametric Mann-Whitney $U$ test was applied to analyze based on the distribution of variables. For correlation analysis, correlations between continuous variables were analyzed through partial correlation analysis accounting for age, gender, and smoking status, or Spearman correlation analysis. Furthermore, multivariable logistic regression analysis was performed to examine the association between the methylation level of $\mathrm{m} 6 \mathrm{~A}$ or the expression of corresponding modulators and clinical symptoms (e.g., AAA rupture). Demographic characteristics and comorbidities were adjusted as confounding factors in all multivariable logistic regression models. Upper and lower strata clinical plasma examination levels were defined according to the cut-off values for the $75^{\text {th }}$ percentile. $\mathrm{P}$ values $\leq 0.05$ were considered statistically significant.

\section{Results}

\section{Characterization of study subjects and histological analysis}

The demographic and clinical characteristics of AAA patients and controls included in this study are shown in Table 2. The youngest AAA subject was 46 years old, and the oldest subject was 74 years old. The mean maximum diameter of AAA was $74.23 \pm 4.44 \mathrm{~mm}$. Twenty-eight percent of AAA patients suffered from a ruptured aneurysm, and $54.8 \%$ were smokers. The youngest control subject was 50 years old, and the oldest subject was 71 years old. The average age of the control group was $58.75 \pm 1.82$ years. None of the control subjects had any obvious atherosclerotic changes within the aortic wall, as assessed by light microscopy. Both study groups were similar in body mass index (BMI) and gender. There were also no significant differences in the prevalence of comorbidities, including DM, hyperlipidemia, carotid diseases, and renal diseases. The symptoms and blood parameters of the AAA patients are summarized in Table 3. 
Table 3 Clinical data of AAA patients

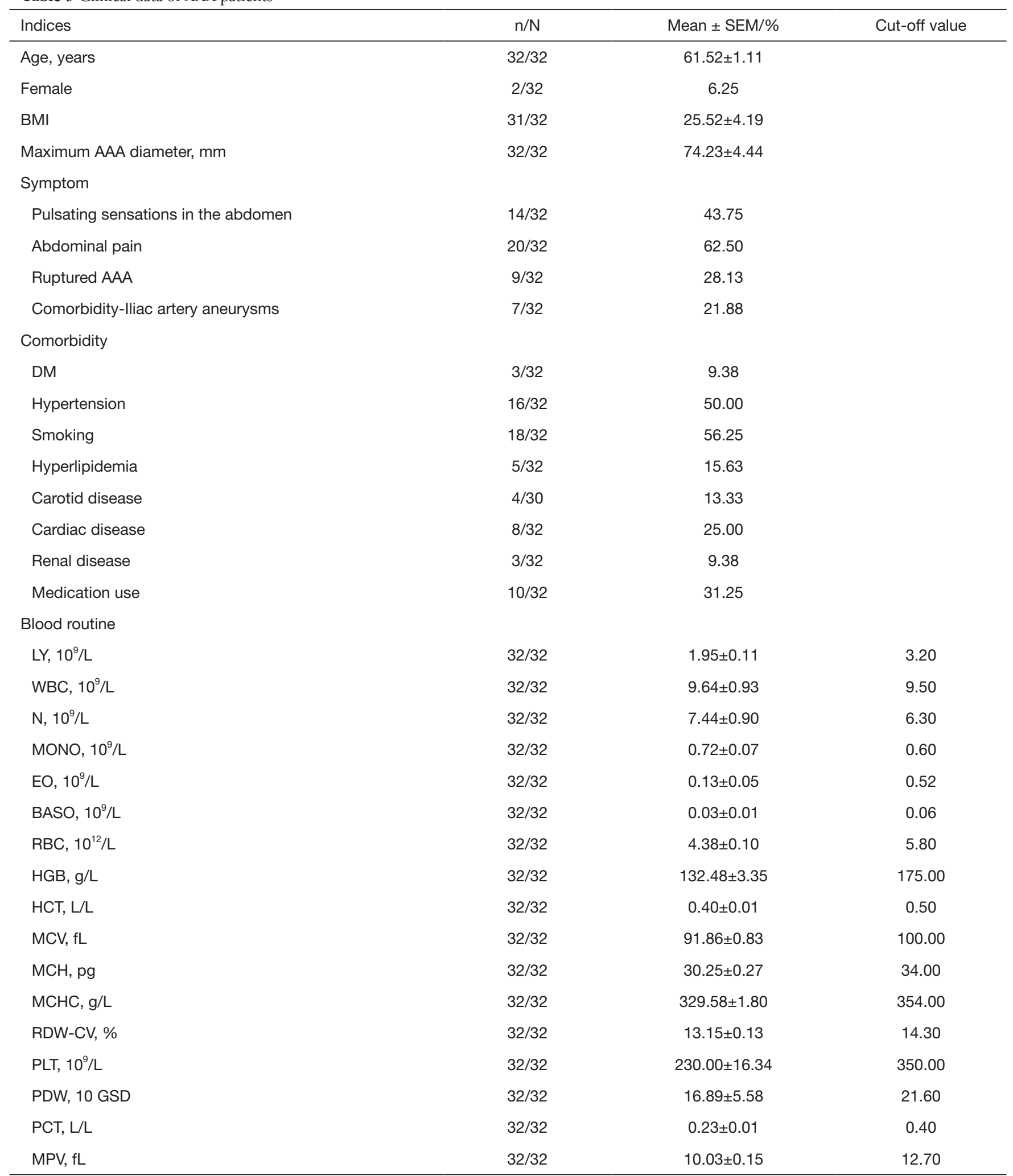

Table 3 (continued) 
Table 3 (continued)

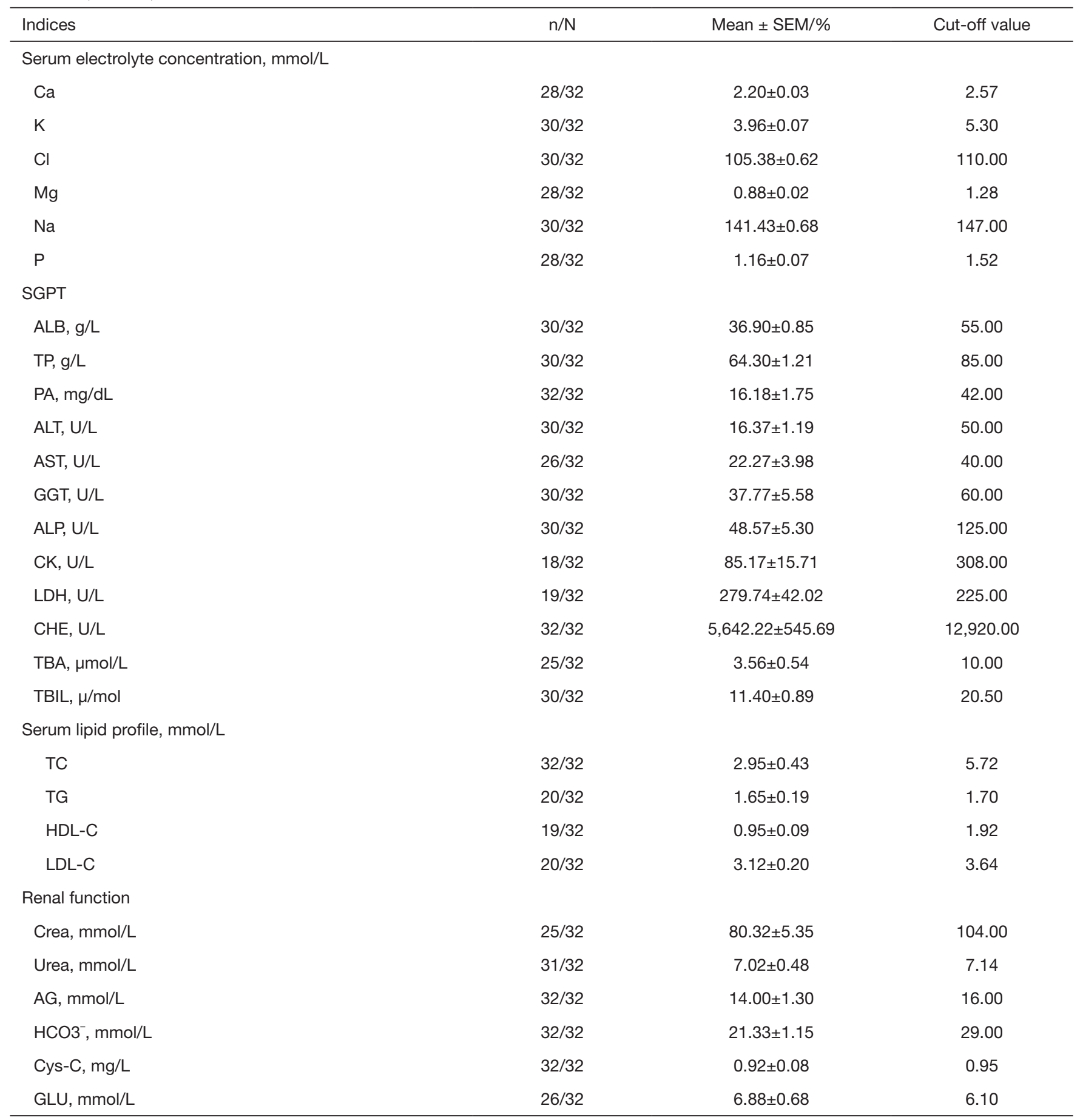

Table 3 (continued) 
Table 3 (continued)

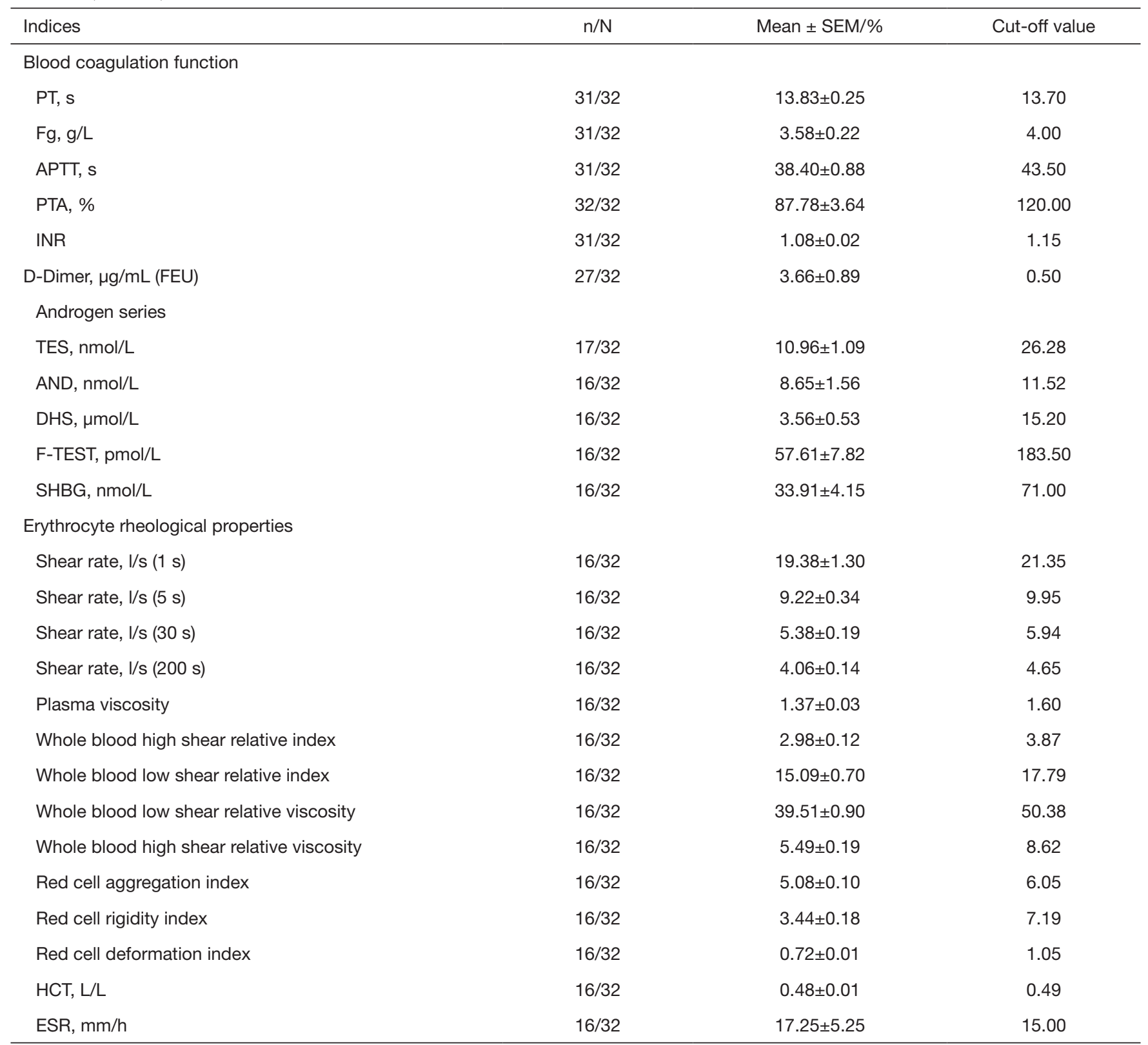

AAA, abdominal aortic aneurysm; SEM, standard error of mean; BMI, body mass index; DM, diabetes mellitus; LY, lymphocyte; WBC, white blood cell; N, neutrophil; MONO, monocyte; EO, eosinophils; BASO, basophil; RBC, red blood cell; HGB, hemoglobin; HCT, hematocrit; MCV, mean corpuscular volume; $\mathrm{MCH}$, mean corpuscular hemoglobin; RDW-CV, red blood cell volume distribution width; $\mathrm{MCHC}$, mean corpuscular hemoglobin concentration; PLT, platelets; PDW, platelet distribution width; PCT, platelet hematocrit; MPV, mean platelet volume; Ca, serum calcium; K, serum kalium; Cl, serum chlorine; Mg, serum magnesium; Na, serum sodium; P, serum phosphate; SGPT, serum glutamic pyruvic transaminase; ALB, albumin; TP, total protein; PA, pre-albumin; ALT, alanine aminotransferase; AST, aspartate aminotransferase; GGT, gamma glutamyl transpeptidase; ALP, alkaline phosphatase; CK, creatine kinase; LDH, lactate dehydrogenase; CHE, cholinesterase; TBA, total bile acid; TBIL, total bilirubin; TC, total cholesterol; TG, triglycerides; HDL-C, highdensity lipoprotein-cholesterol; LDL-C, low-density lipoprotein-cholesterol; Crea, creatinine; AG, anion gap; Cys-C, cystatin C; GLU, fasting plasma glucose; PT, prothrombin time; APTT, activated partial thromboplastin time; PTA, prothrombin activity; INR, international normalized ratio; TES, testosterone; AND, androgen; SHBG, sex hormone-binding globulin; DHS, dehydroepiandrosterone; F-TEST, free testosterone; ESR, erythrocyte sedimentation rate. 
A semi-quantitative histological characterization of all AAA tissue samples was performed to assess the extension of the individual histopathological features in the AAA wall (Table 4). Furthermore, to compare the histopathological features of AAA with those of healthy aortas, semi-quantitative scoring was also performed for the control group. Because control tissue samples showed the same scoring results, Table 4 shows only one example of the histological characterization of the control group [healthy aorta control (Ctrl)]. Furthermore, IHC was used to differentiate between the four main cell types in atherosclerotic lesions and AAAs (ECs, lymphocytes, macrophages, and SMCs).

\section{Increased m6A mRNA methylation level occurred in $A A A$ tissue samples}

The relative m6A mRNA methylation status in AAA tissue samples was significantly associated with the higher percentage of $\mathrm{m} 6 \mathrm{~A}$ in total $\mathrm{mRNA}$ compared with the control aortic tissue samples (more than 2-fold; $\mathrm{P}=0.007$; Figure $2 A$ ). Moreover, using a multivariable model for analysis, higher m6A methylation levels represented an even greater risk of AAA rupture when compared with nonruptured AAAs [odds ratio (OR), 1.81; 95\% confidence interval (CI), 1.370 (1.007, 1.870); $\mathrm{P}=0.047]$. In AAA patients with an iliac aortic aneurysm, the adjusted OR of the m6A methylation level was increased to 1.238 (1.020 to 1.502; $\mathrm{P}=0.031)$ as compared to that of AAA patients only (Table 5).

\section{The $m R N A$ expression of $m 6 A$ modulators in $A A A$ tissue samples}

We then determined the mRNA expression levels of several molecules participating in m6A mRNA modification. The expression levels of YTHDF2 and YTHDF3 were significantly up-regulated, while FTO expression was significantly down-regulated in AAA tissue samples compared with controls. There was no significant difference in the expression levels of the other genes, including METTL3, METTL14, ALKBH5, and YTHDF1 (Figure 2B,C,D,E,F,G,H), although there was a trend toward down-regulation for each of these. High mRNA expression of YTHDF2 and YTHDF3 represented an even greater risk of AAA rupture as compared to non-ruptured AAAs (YTHDF2, OR, 1.151; 95\% CI, 1.006-1.318; $\mathrm{P}=0.041$; YTHDF3, OR, 1.036; 95\% CI, 1.001-1.072; $\mathrm{P}=0.044)$ in model 3 (Table 6).

\section{The correlations among the $m R N A$ expression levels of m6A modulators in AAA tissue samples}

We next analyzed the correlations among relative mRNA expression levels. METTL3 expression significantly was correlated with METTL14, YTHDF1, YTHDF2, and YTHDF3 expression $(\mathrm{R}=0.694,0.959,0.927$, and 0.960 , respectively; $\mathrm{P}<0.001$ for all comparisons). METTL 14 expression was significantly correlated with YTHDF1, YTHDF2, and YTHDF3 ( $\mathrm{R}=0.672,0.679$, and 0.793, respectively; $\mathrm{P}<0.001, \mathrm{P}<0.001$, and $\mathrm{P}<0.001$, respectively). ALKBH5 expression was significantly correlated with YTHDF2 ( $\mathrm{R}=0.433$, and $\mathrm{P}=0.044)$. YTHDF1 expression was significantly correlated with YTHDF2 and YTHDF3 $(\mathrm{R}=0.840$ and 0.905 , respectively; $\mathrm{P}<0.001$ and $\mathrm{P}<0.001$, respectively). YTHDF2 expression was significantly correlated with YTHDF3 $(\mathrm{R}=0.913$ and $\mathrm{P}<0.001)$ (Table 7).

\section{The level of $m 6 A$ mRNA methylation was correlated with the $m R N A$ expression levels of $m 6 A$ modulators in $A A A$ tissue samples}

The m6A\% in total mRNA significantly was correlated with METTL3, METTL14, YTHDF1, YTHDF2, and YTHDF3 expression levels $(\mathrm{R}=0.483,0.489,0.541$, 0.479 , and 0.559 respectively; $\mathrm{P}=0.023, \mathrm{P}=0.021, \mathrm{P}=0.009$, $\mathrm{P}=0.024$, and $\mathrm{P}=0.007$, respectively; Table 7).

\section{Increased protein expression levels of m6A modulators between $A A A$ and bealthy aorta}

Given the mRNA expression levels of m6A modulators and their interrelatedness in human AAA tissue samples, we selectively performed western blot analysis for representative RNA methyltransferases, demethylases, and m6A binding proteins, including METTL14, FTO, YTHDF1, and YTHDF3. In Figure 3, the protein expression levels of METTL14, FTO, YTHDF1, and YTHDF3 in AAA tissue samples were significantly increased compared with those in healthy control aortic tissue samples $(\mathrm{P}<0.001, \mathrm{P}<0.001$, $\mathrm{P}<0.001$, and $\mathrm{P}<0.001$, respectively).

\section{Increased protein expression levels of $m 6 A$ modulators in AAA tissue samples and their cellular locations}

Subsequently, we examined the localization of METTL14, 
Table 4 Histological characterization of all AAA tissue samples

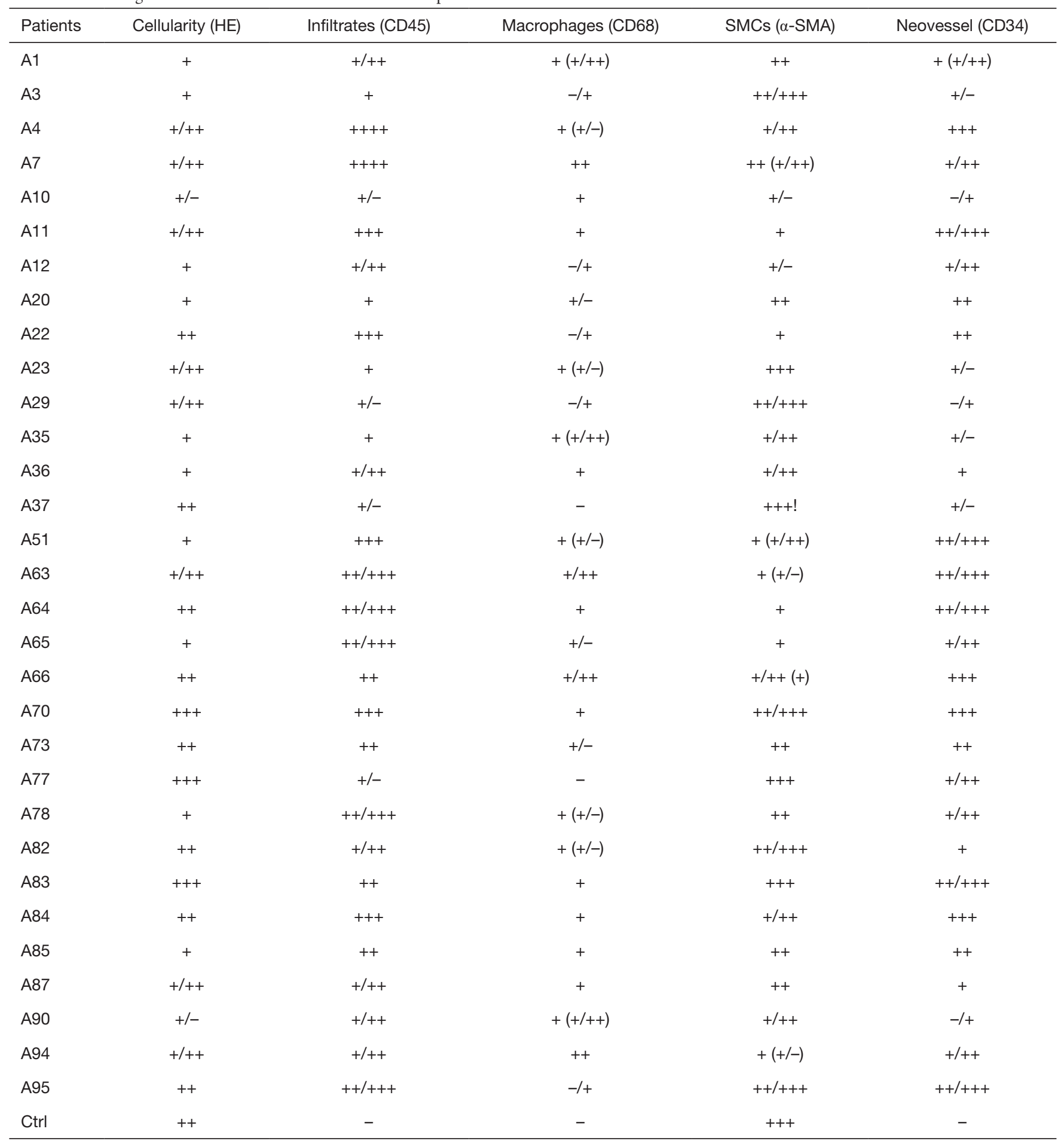

A, abdominal aortic aneurysm; Ctrl, healthy aorta control; AAA, abdominal aortic aneurysm; HE, hematoxylin-eosin; SMC, smooth muscle cell. 


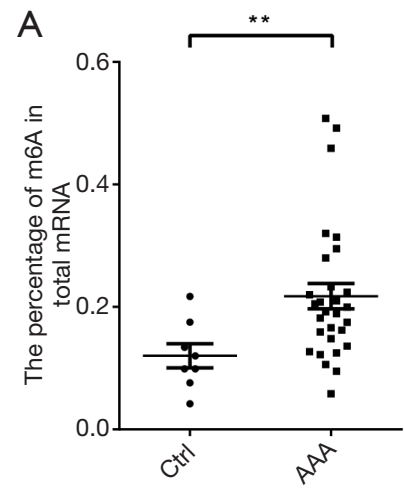

E

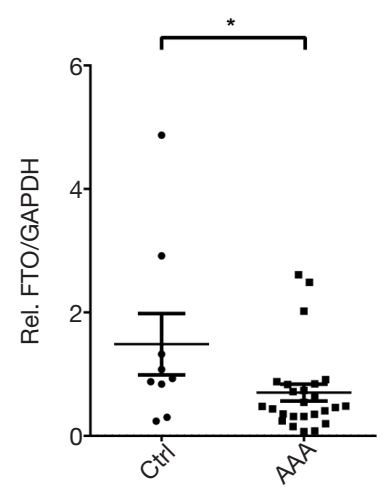

B

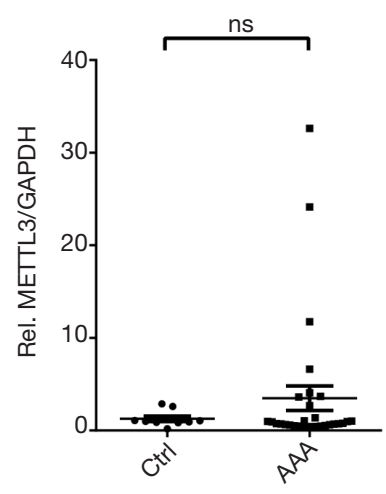

F

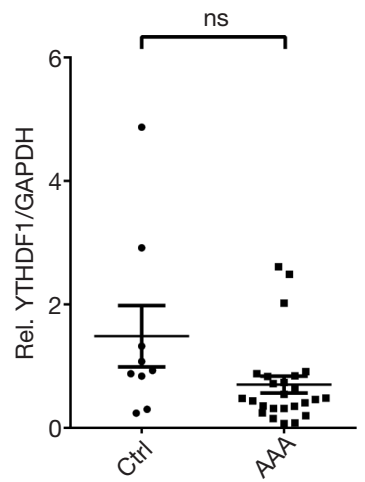

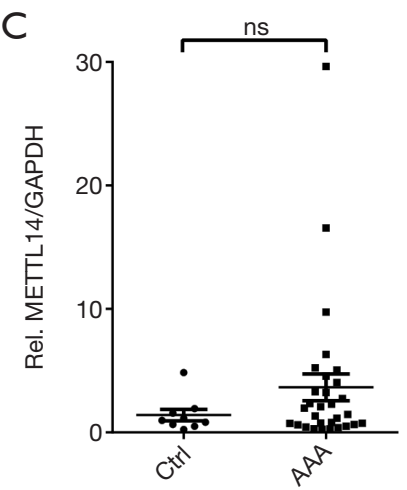

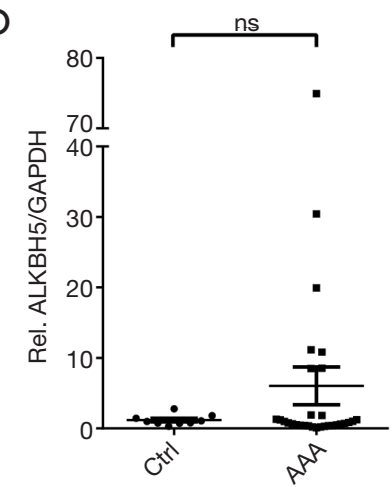

$\mathrm{H}$

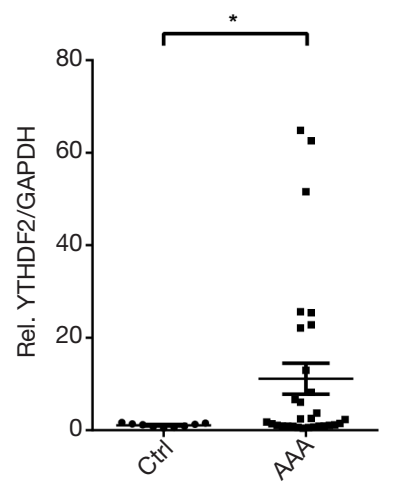

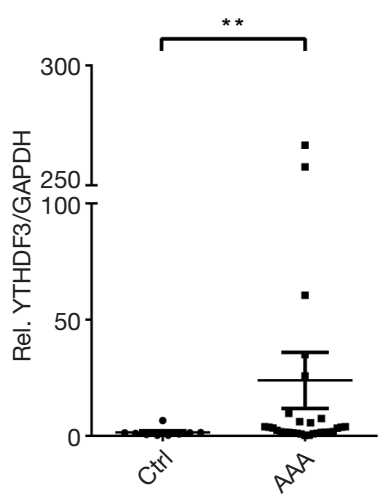

Figure 2 Expression of m6A RNA methylation status (A) and m6A methylation modulators (B,C,D,E,F,G,H) at the mRNA level in AAA tissue samples compared with the healthy control aortas, analyzed by qRT-PCR and SYBR Master Mix. The non-parametric Mann-Whitney U test was applied to analyze the mRNA methylation status (A); the m6A "writers" family, METTL3 (B) and METTL14 (C); m6A "erasers" family, ALBH5 (D) and FTO (E); and "readers" family, YTHDF 1 (F), YTHDF2 (G), and YTHDF3 (H). Dates are normalized to the expression of GAPDH and analyzed through the non-parametric Mann-Whitney $\mathrm{U}$ test. *, $\mathrm{P}<0.05$; **, $\mathrm{P}<0.01$. m6A, N6-methyladenosine; AAA, abdominal aortic aneurysm; qRT-PCR, quantitative real-time polymerase chain reaction; ns, not significant.

FTO, and YTHDF3, using IHC in consecutive sections. METTL14 staining was found to colocalize with CD45+ leukocytes and CD34+ ECs. YTHDF3 was found to colocalize with CD68+ macrophages and SMCs, and weakly with CD34+ ECs. Finally, we observed that FTO was colocalized with CD3+ T lymphocytes and SMCs and weakly with CD34+ ECs (Figure 4).

The semi-quantitative histological characterization and the results of RT-PCR were analyzed again by calculating the correlation coefficient to explore any relationships between mRNA level and the morphology within AAA. Three significantly positive correlations between the histological and immunohistochemical classification and
mRNA level expression of m6A methylase were observed. First, neovascularization was positively associated with the mRNA level of METTL14 $(\mathrm{R}=0.487, \mathrm{P}=0.007)$. Furthermore, a significant positive correlation was found between the amount of smooth muscle cells and the expression of FTO $(\mathrm{R}=0.425, \mathrm{P}=0.027)$. We also found a significant positive correlation between the amount of macrophage infiltrates and the expression of YTHDF3 ( $\mathrm{R}=0.531, \mathrm{P}=0.001)$. Beyond that, no significant correlation was detected between the other histological assessments and the expression of m6A modulators (Table 8).

In contrast to AAA tissue samples, no protein expression was detected for METTL14, FTO, or YTHDF3 in the 


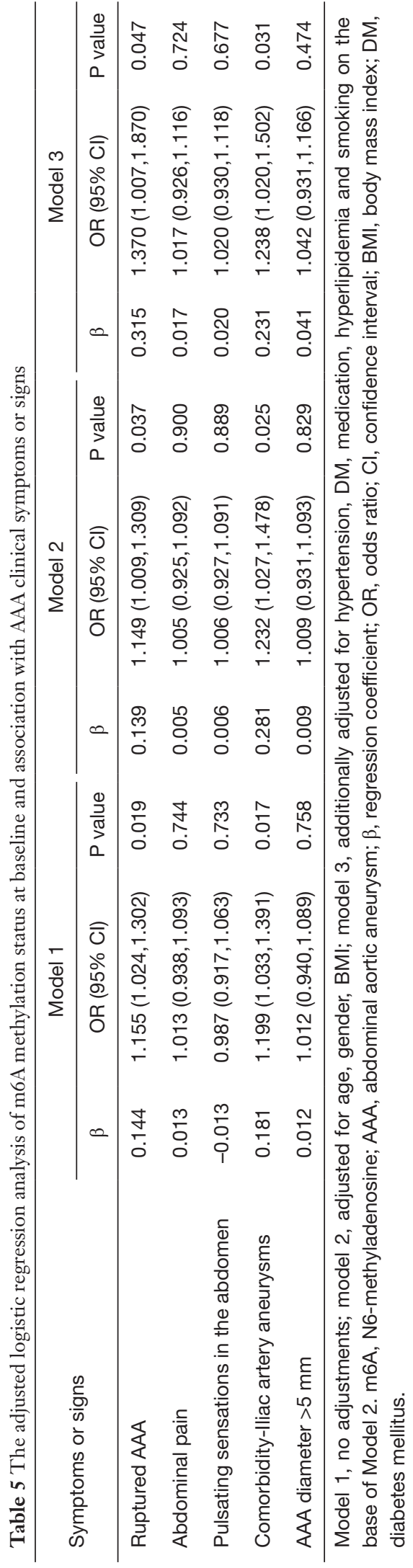

healthy aortic tissue samples. Representative images of these results are provided in Figure 5.

\section{$m 6 A$ mRNA methylation, expression levels of m6A modulators and association with clinical data}

We evaluated the possible correlations between m6A\% in AAA tissue and clinical data. Interestingly, m6A\% was found to correlate with AAA diameter $(\mathrm{R}=0.534 ; \mathrm{P}=0.002)$ positively. Additionally, m6A\% also significantly correlated with serum $\mathrm{LDH}$ concentration $(\mathrm{R}=0.329 ; \mathrm{P}=0.004)$. After multivariate analysis of patients with larger AAA diameter, the adjusted OR of the m6A methylation level was increased to 1.20 (1.03 to $1.39 ; \mathrm{P}=0.019)$ than patients with smaller AAA diameter.

We next analyzed the correlations between the expression levels of the m6A modulators and clinical parameters. In agreement with the above-described findings, METTL3 expression positively correlated to AAA diameter $(\mathrm{R}=0.406$, $\mathrm{P}=0.029)$. Moreover, METTL3 expression also correlated to activated partial thromboplastin time (APTT) and erythrocyte sedimentation rate (ESR) $(\mathrm{R}=0.368$ and 0.523 , respectively, and $\mathrm{P}=0.049$ and $\mathrm{P}=0.038$, respectively). METTL14 expression significantly correlated to lymphocyte (LY), white blood cell (WBC), serum sodium level and plasma alkaline phosphatase $(\mathrm{R}=0.389,0.437$, -0.412 , and -0.445 , respectively; $\mathrm{P}=0.037, \mathrm{P}=0.018$, $\mathrm{P}=0.030$, and $\mathrm{P}=0.018$, respectively). ALKBH5 expression was significantly correlated to $\mathrm{MONO}$ and gamma glutamyl transpeptidase (GGT) $(\mathrm{R}=-0.384$ and 0.435 , respectively; $\mathrm{P}=0.040$ and $\mathrm{P}=0.021$, respectively). YTHDF1 expression was significantly correlated to AAA diameter and plasma viscosity $(\mathrm{R}=0.549$ and 0.507 , respectively; $\mathrm{P}=0.002$ and $\mathrm{P}=0.045$, respectively). YTHDF2 expression was significantly correlated to MONO, AST, and CHE $(\mathrm{R}=-0.435,-0.468$ and 0.374 , respectively; $\mathrm{P}=0.018$, $\mathrm{P}=0.018$, and $\mathrm{P}=0.042$, respectively). YTHDF3 expression significantly correlated to AAA diameter, MONO count, and GGT $(\mathrm{R}=0.370,-0.483$, and 0.379 , respectively; $\mathrm{P}=0.048, \mathrm{P}=0.008$, and $\mathrm{P}=0.040$, respectively).

\section{Discussion}

The m6A modification of mRNA is a crucial posttranscriptional regulation for various fundamental bioprocesses and has a critical impact on the splicing, exporting, and translation of mRNA (28). M6A modification also influences mRNA stability. Mathiyalagan et al. have 


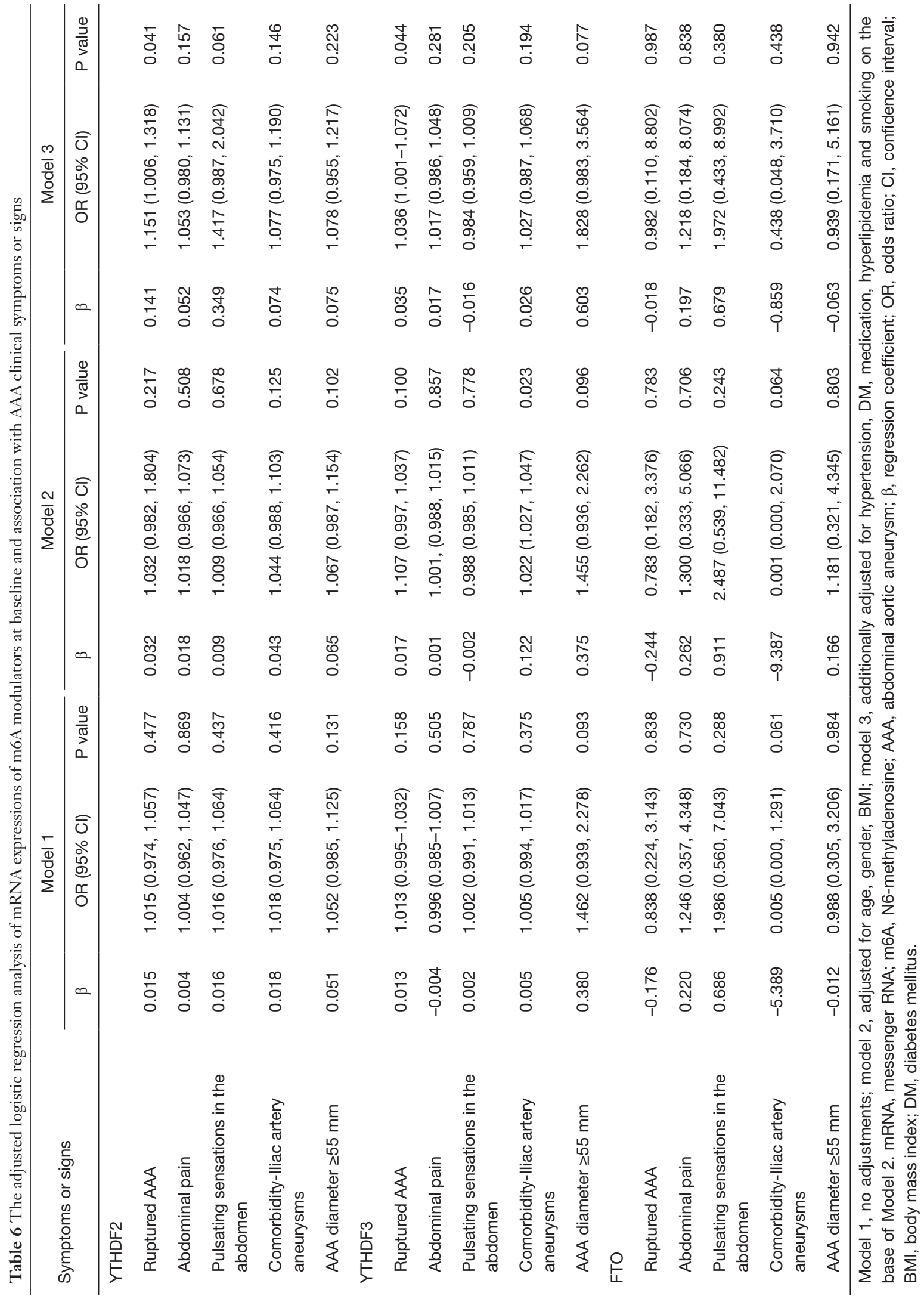


Table 7 Correlation among mRNA m6A\% and the mRNA expressions of m6A modulators

\begin{tabular}{|c|c|c|c|c|c|c|c|c|}
\hline Partial correlations & METTL3 & METTL14 & ALKBH & FTO & YTHDF1 & YTHDF2 & YTHDF3 & $\mathrm{m} 6 \mathrm{~A}(\%)$ \\
\hline Partial correlation coefficient & - & & & & & & & \\
\hline$P$ value & - & & & & & & & \\
\hline \multicolumn{9}{|l|}{ METTL14 } \\
\hline$P$ value & 0.000 & - & & & & & & \\
\hline \multicolumn{9}{|l|}{ ALKBH } \\
\hline Partial correlation coefficient & 0.205 & -0.084 & - & & & & & \\
\hline$P$ value & 0.360 & 0.710 & - & & & & & \\
\hline$P$ value & 0.124 & 0.194 & 0.394 & - & & & & \\
\hline \multicolumn{9}{|l|}{ YTHDF1 } \\
\hline Partial correlation coefficient & 0.959 & 0.672 & 0.138 & -0.395 & - & & & \\
\hline$P$ value & 0.000 & 0.001 & 0.539 & 0.068 & - & & & \\
\hline \multicolumn{9}{|l|}{ YTHDF2 } \\
\hline Partial correlation coefficient & 0.927 & 0.679 & 0.433 & -0.297 & 0.840 & - & & \\
\hline$P$ value & 0.000 & 0.001 & 0.044 & 0.179 & 0.000 & - & & \\
\hline \multicolumn{9}{|l|}{ YTHDF3 } \\
\hline
\end{tabular}

Controlled with age, gender, and smoking. mRNA, messenger RNA; m6A, N6-methyladenosine.

reported that $\mathrm{m} 6 \mathrm{~A} \mathrm{mRNA}$ modification also influences cardiac function (29).

However, studies of m6A in the cardiovascular field are still lacking. In this study, our team were the first to focus on m6A modification of mRNA in AAA and to demonstrate the critical impact of the modification on the progression of AAA. In this research, we first demonstrated that in AAA tissue samples there was an increasing percentage of mRNA modified by $\mathrm{m} 6 \mathrm{~A}$ relative to healthy aortic samples.

To detect the possible effectors participating in m6A modification, we subsequently analyzed the mRNA expression of several important and relevant proteins.
Significant upregulations were observed in the mRNA expression levels of YTHDF2 and YTHDF3, while significant downregulation was found for FTO. FTO mediates the demethylation of $\mathrm{m} 6 \mathrm{~A}$ in the poly(A) tail of mRNA and thus influences the splicing of pre-mRNA (28). YTHDF2 promotes the degradation of $\mathrm{m} 6 \mathrm{~A}$-methylated mRNA (30), while YTHDF3 is thought to influence the metabolism of m6A-methylated mRNA (31). In addition, we found significant correlations among $\mathrm{m} 6 \mathrm{~A} \%$ and the mRNA expression levels of these modulators, including "writers" (METTL14 and METTL3), "erasers" (FTO and ALKBH5), and "readers" (YTHDF1, YTHDF2, 
A
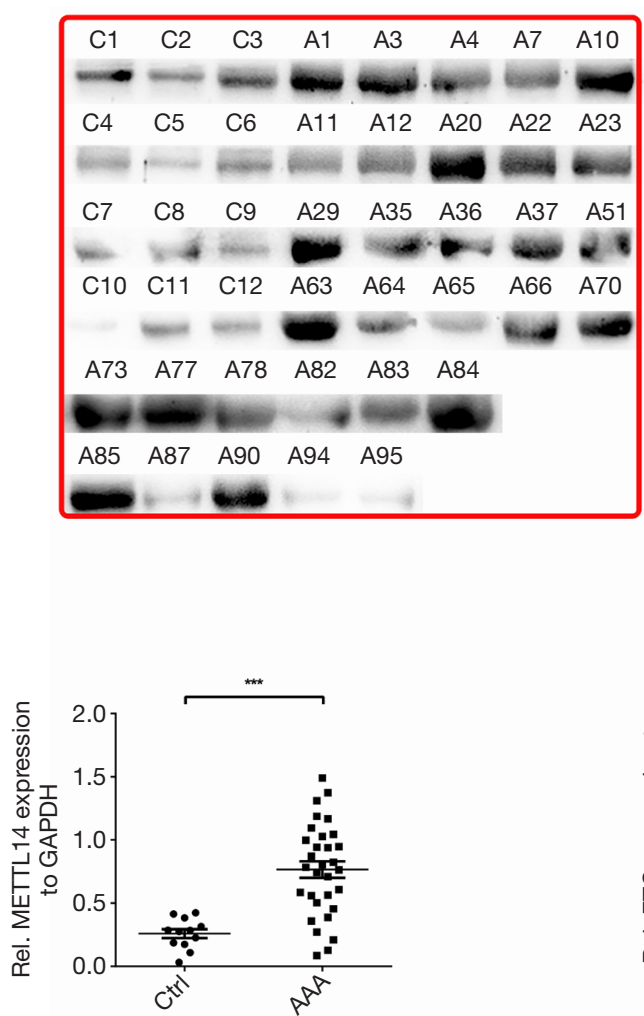

C
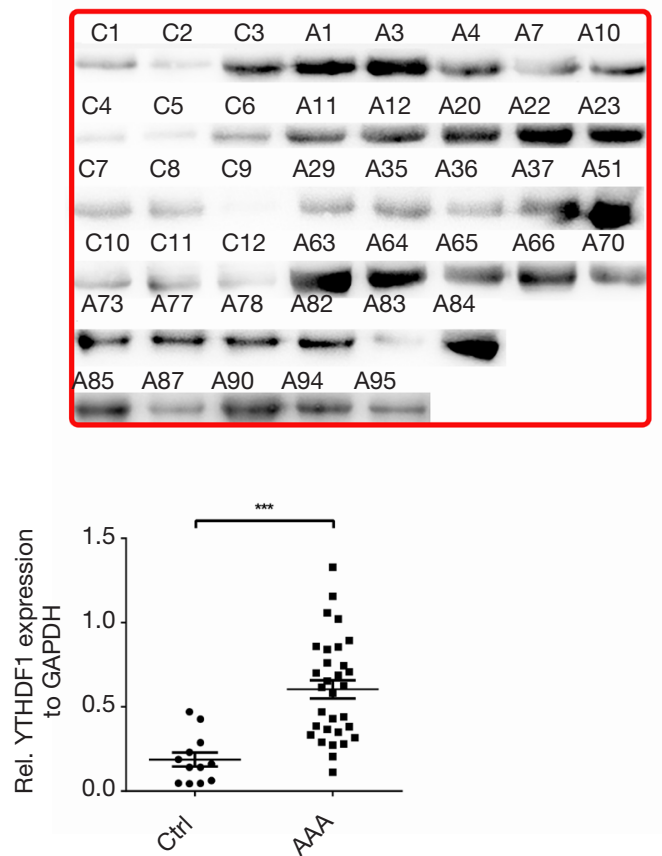

$B$
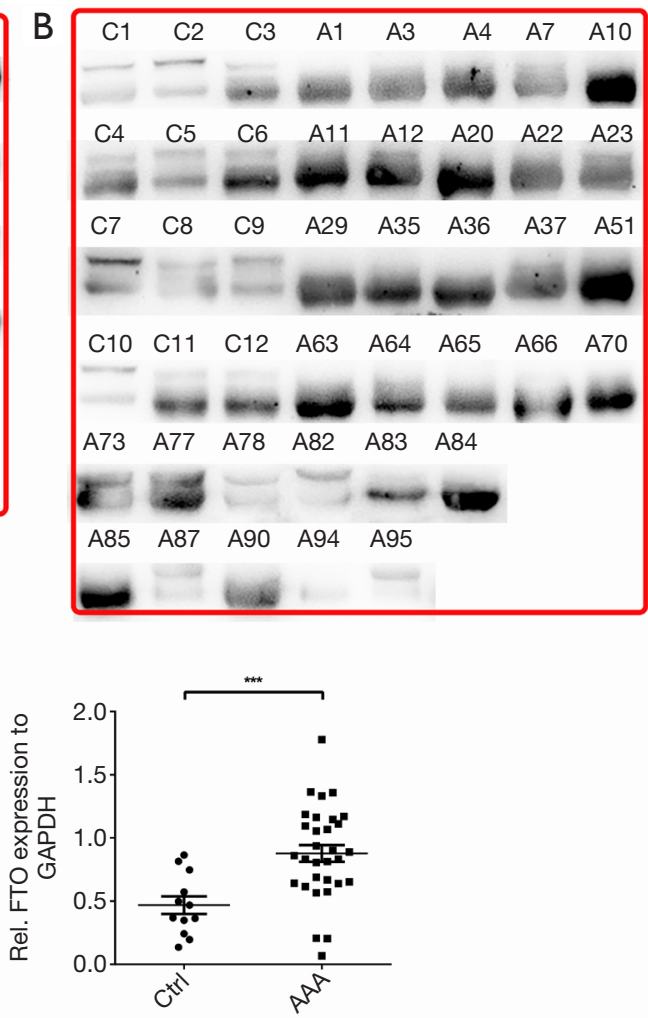

D

Figure 3 Expression analysis of METTL14 (A), FTO (B), YTHDF1 (C), and YTHDF3 (D) in AAA and healthy aortas at the protein level through the non-parametric Mann-Whitney $\mathrm{U}$ test. At top are the raw images of blots by Western blot, and at bottom is quantification of the band intensities relative to the expression of GAPDH. ${ }^{* * *}, \mathrm{P}<0.001$. C and Ctrl, control healthy aorta $(\mathrm{n}=12)$; A and AAA, specimens of abdominal aortic aneurysm $(\mathrm{n}=32)$. 
A

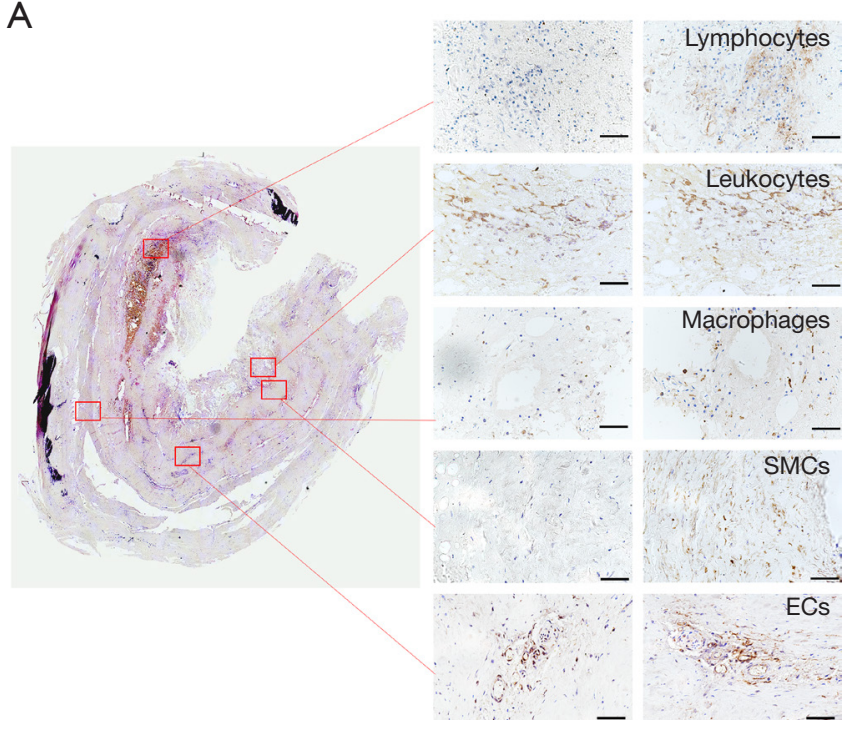

B

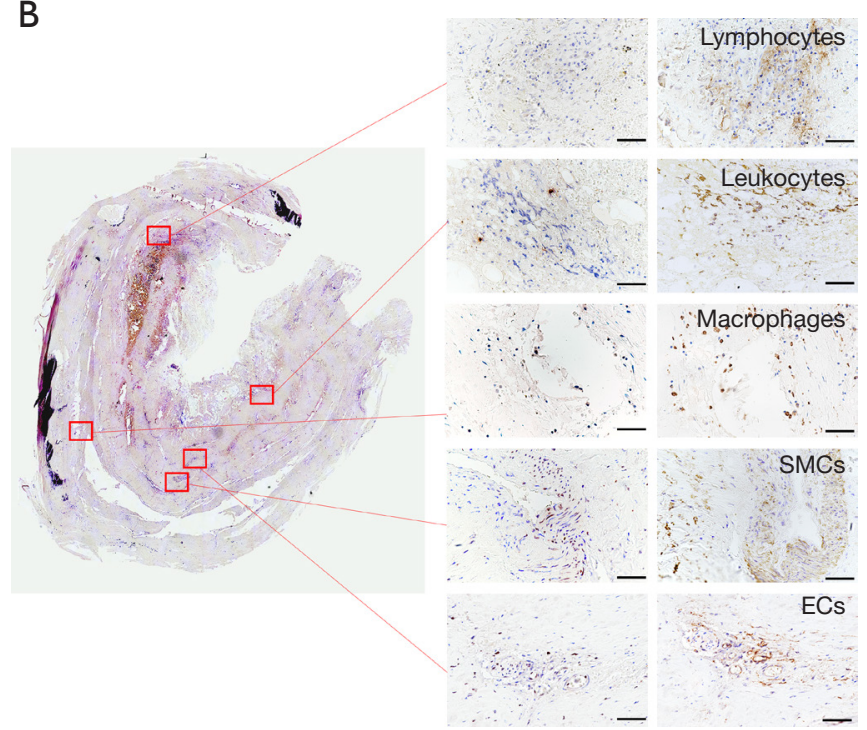

\section{C}

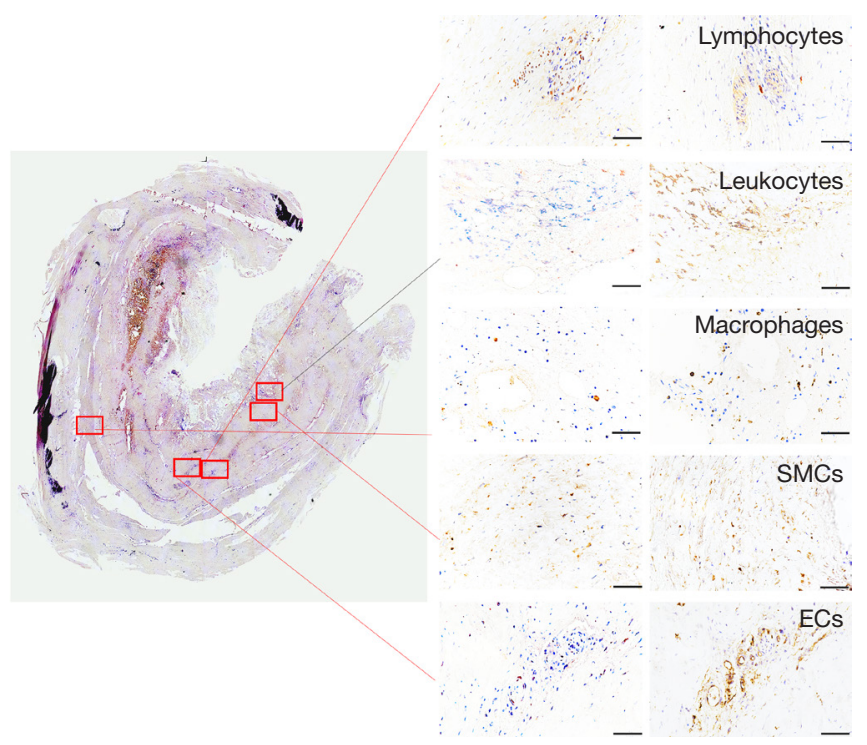

Figure 4 Analysis of METTL14 (A), FTO (B), and YTHDF3 (C) in AAA using IHC for cellular localization. Overview image (left panel) of the whole AAA tissue sample with areas selected for cellular localization of m6A modulator expressions (HE staining). The magnified images depict consecutive staining of individual cell types, as revealed by staining for indicated markers within the AAA wall and indicated corresponding substrates. Scale bar, $50 \mu \mathrm{m}$. AAA, abdominal aortic aneurysm; IHC, immunohistochemistry; m6A, N6-methyladenosine; HE, hematoxylin-eosin; SMC, smooth muscle cell; EC, endothelial cell.

and YTHDF3). The mRNA m6A modification is a tight, complex, and abundant post-transcriptional modification. The correlations indicate that crosstalk among writers, readers, and erasers may have the potential functional importance for regulating AAA progression.

Subsequently, we determined the expression levels of
METTL14, FTO, YTHDF1, and YTHDF3 at the protein level. METTL3, METTL14, and WTAP constitute the RNA methyltransferase complex (9), which catalyzes m6A modification reactions. Previous studies on cancer and stem cells have reported that the deletion of METTL14 selectively downregulates overall m6A levels in m6A- 
Table 8 Correlations between mRNA expressions of m6A modulators and histology of AAAs

\begin{tabular}{lccccc}
\hline $\mathrm{R}$ & Infiltrates (CD45) & Infiltrates (CD34) & Macrophages (CD68) & SMCs $(\alpha$-SMA) & Neovessel (CD34) \\
\hline METTL14 & 0.147 & 0.095 & 0.089 & -0.098 & $0.487^{\star \star}$ \\
FTO & 0.105 & -0.144 & -0.246 & $0.425^{\star}$ & 0.186 \\
YTHDF3 & 0.295 & -0.007 & $0.531^{\star \star}$ & 0.145 & 0.117 \\
\hline
\end{tabular}

*, $\mathrm{P}<0.05 ;{ }^{* \star}, \mathrm{P}<0.01$. mRNA, messenger RNA; m6A, N6-methyladenosine; AAA, abdominal aortic aneurysm; SMC, smooth muscle cell.

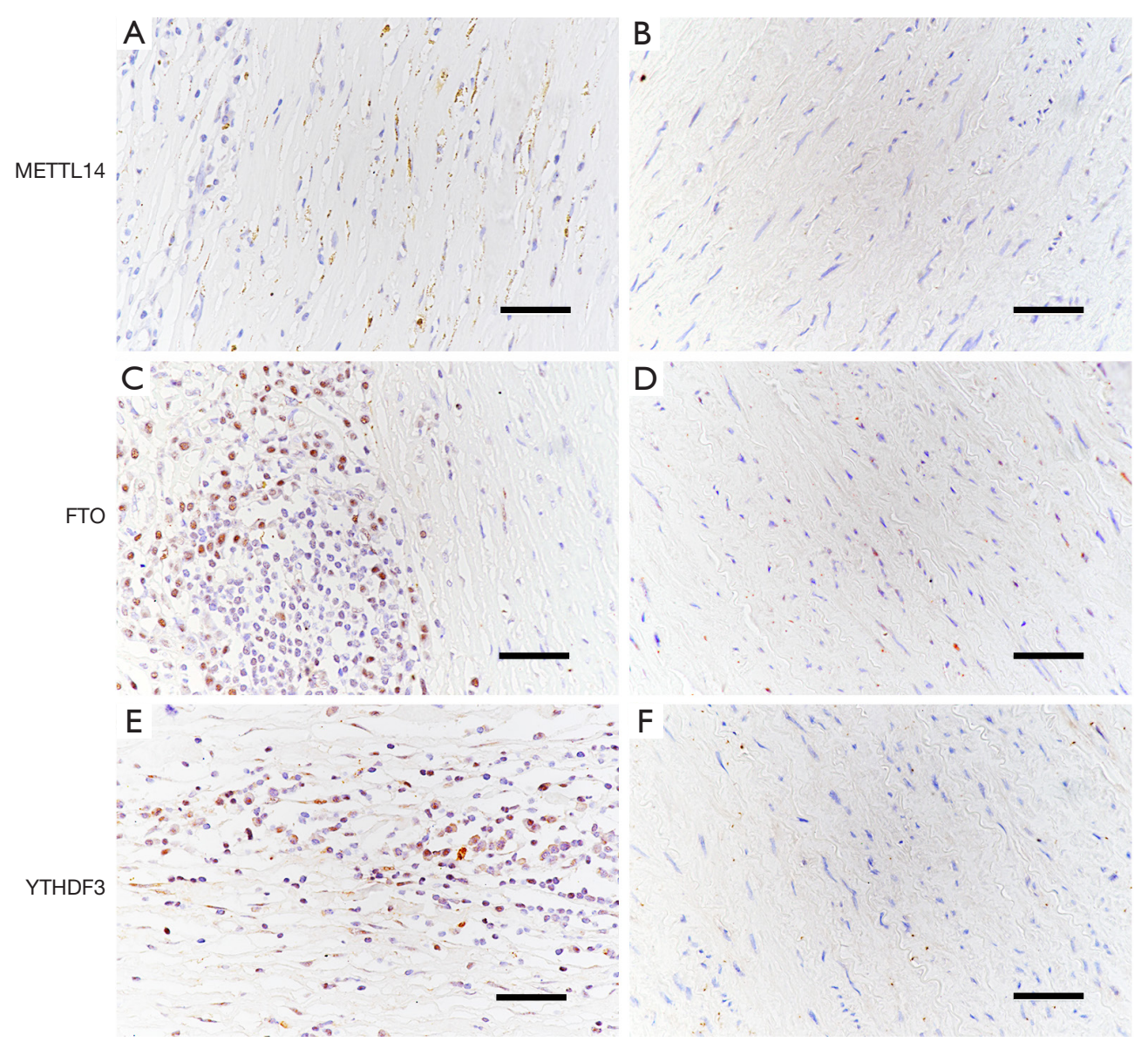

Figure 5 Analysis of acetylated METTL14 (A), FTO (C), and YTHDF3 (E) in AAA using IHC, compared with their expressions in healthy control aortic tissues $(\mathrm{B}, \mathrm{D}, \mathrm{F})$. Scale bar, $50 \mu \mathrm{m}$. AAA, abdominal aortic aneurysm; IHC, immunohistochemistry.

methylated mRNA (9), which demonstrates the critical biological functions of METTL14.

In the present study, we found that METTL14 expression had increased in AAA tissues. This evidence may show there to be a potential impact of METTL14 on the development of AAA. FTO is a notable demethylase that represses the stability of m6A-modified mRNA, and it was upregulated at the protein level in the AAA tissue samples.
YTHDF1 is known to be an important m6A reader that promotes the translation of m6A-modified mRNA (32). High expression levels of YTHDF1 and YTHDF3 may enhance translation efficiency and regulate the metabolism of mRNA in AAA. The upregulation of these modulators in AAA indicates complex m6A modifications in AAA tissue. According to the results of m6A mRNA immunoprecipitation in previous studies, different $\mathrm{m} 6 \mathrm{~A}$ 
modulators may target different mRNAs $(19,33)$, which may again contribute to the differences among gene expression levels. The same situation seems to appear also in AAA. Therefore, we propose the hypothesis that m6A methylation may play a key role in the development of AAA in a way similar to its biological function in cancers and other pathological processes.

The results of IHC analysis for the expressions and localization of these proteins in AAA provide new insight for the potential role of m6A in the progression of AAA. In the present study, the differences among the expressions of METTL14, FTO, and YTHDF3 were found in the different types of inflammatory cells that exist in the AAA tissues. For one, inflammatory cells regulating the AAA development and being involved in the pathogenesis of AAA has been reported (5,6,34-36). For another, previous studies have provided evidence that $\mathrm{m} 6 \mathrm{~A}$ mRNA modification can regulate $\mathrm{T}$ cell homeostasis (37) and the inflammatory response of human dental pulp cells (24). Based on these findings, we surmise that m6A modification may have a crucial impact on the expressions of genes in different types of inflammatory cells, which cooperatively regulate AAA progression.

We also found that the levels of the expression of m6A modulators were different in CD34+ ECs. Angiogenesis is a critical mechanism for the development of AAA. Recently, researchers found a trend toward of decreased m6A and METTL14 in hepatocellular carcinoma (16); however, the role of m6A in hepatocellular carcinoma remains unknown. The authors also hypothesized that miRNA-126 might inhibit the repressive effect of METTL14 on tumor metastasis. A recent study demonstrated that miR-126 contributes to endothelial permeability and apoptosis (38). In the present study, we indeed found that METTL14 was colocalized to smooth muscle cells and CD34+ ECs. Therefore, we hypothesize METTL14 might play a significant role in AAA by regulating EC function. Finally, we detected the expression of these modulators in SMCs, as $\mathrm{ECM}$ degradation in AAA is relevant to SMC apoptosis. m6A methylation has potential functions in a variety of pathogenic processes that are relevant in AAA progression, including apoptosis, cell proliferation, and matrix degradation.

Expression analyses showed that YTHDF3 mRNA and protein levels were significantly increased in the vessel walls of AAA patients as compared to healthy aortas. Our findings are biologically plausible for several reasons. First, high expression of YTHDF3 was previously found in inflammatory cells, like CD3+ lymphocytes, in the diseased aorta. Our results also showed there to be a significant correlation between the expression of YTHDF3 and the lymphocyte marker CD3. AAA is characterized by chronic inflammation in the tunica media and adventitia, which leads to the upregulation and release of multiple cytokines, the activation of a plethora of proteolytic enzymes, and ultimately to a rapid expansion of AAA and rupture (39). Logistic regression analysis revealed that increased expression of YTHDF3 is an even greater risk for AAA rupture. In addition, our results demonstrate that the expression of YTHDF3 is significantly associated with the diameter of AAA. This is an important finding, as YTHDF3 is the main switch for causing inflammation, which again is a driving force in AAA progression. Consequently, YTHDF3 may also be a potential biological marker for patients who are at increased risk of AAA rupture. Finally, it is important to mention that the expression of YTHDF3 was also positively correlated with the abundance of mononuclear leucocytes, and it is already well known that inflammatory cells play a crucial role during the development and progression of AAA.

In our present study, m6A methylation provided new insights into the pathogenesis of AAA. However, the target genes of m6A methylation in AAA remain unclear. Therefore, further research should be focused on m6A target genes in AAA, and this may contribute to the development of the clinical application of molecule-targeted treatment of AAA.

There are some limitations to our current study which should be addressed. Our study includes a small sample size. Furthermore, there is a large variation among the values from the individual tissue specimens which were observed. For this reason, we tried to adjust our data by the total amount of cells within the AAA wall, the extent of inflammation, patient age, AAA diameter, hyperlipidemia, smoking history, and rupture. Nevertheless, no significant correlation between the factors used for the adjustment or the expression of m6A modulators was found, and no improvement of our results was achieved.

Furthermore, as most of our samples were formalin-fixed, the cellular localization of m6A methyltransferases was evaluated in consecutively stained sections and indirectly by correlation analyses with specific cell markers. In addition, the analysis of epigenetic changes in inflammatory cells in AAA could not be directly compared with the control of healthy aortic tissue samples, because these specimens do not have many inflammatory cells. Thus, the conclusion that the overexpression of $\mathrm{m} 6 \mathrm{~A}$ modulators is found in CD45 and CD3 positive cells is based on our results in AAA 
alone without any comparison with other inflammatory cells, such as those from the peripheral blood.

\section{Conclusions}

Our study provides the first piece of evidence to explain the significant increase of m6A levels in AAA (Figure S1). Furthermore, the higher expression level of m6A was correlated with an increased risk of rupture in AAA patients. Additionally, the expression of the main modulators involved in $\mathrm{m} 6 \mathrm{~A}$ modification and their inner correlations offer a novel view on the mechanism of m6A modification in the pathogenesis of AAA. However, it is still unclear whether m6A modification through regulating the metabolism and stability of mRNA should be considered a protective or a harmful factor in AAA. Further studies are needed to understand the mechanism in terms of to what extent m6A modification influences AAA progression or the potential increase of rupture risk. As natural or designed compounds can alter enzymatic epigenetic regulators, targeting these molecules may emerge as a potential novel diagnostic and therapeutic strategy in aneurysm diseases.

\section{Acknowledgments}

We thank AME Editing Service (AME Publishing Company) for editing the English text of a draft of the manuscript.

Funding: This work was supported by the National Natural Science Foundation of China (grant number: 81600370), the China Postdoctoral Science Foundation (grant number: 2018M640270), the Natural Science Foundation of Liaoning Province (2019-ZD-0789), and the Basic Research Program of Liaoning Provincial Institutions of Higher Learning 2017 (LZDK201701).

\section{Footnote}

Conflicts of Interest: The authors have no conflicts of interest to declare.

Ethical Statement: The authors are responsible for all aspects of the work in ensuring that questions related to the accuracy or integrity of any part of the work are appropriately investigated and resolved. Participants gave written informed consent before the study, and the study protocol was approved by the Ethics Committee of Shengjing Hospital of China Medical University (CMU) (ethical approval number: 2016PS085K), in accordance with the Declaration of Helsinki.

\section{References}

1. Hirsch AT, Haskal ZJ, Hertzer NR, et al. ACC/ AHA 2005 Practice Guidelines for the management of patients with peripheral arterial disease (lower extremity, renal, mesenteric, and abdominal aortic): a collaborative report from the American Association for Vascular Surgery/Society for Vascular Surgery, Society for Cardiovascular Angiography and Interventions, Society for Vascular Medicine and Biology, Society of Interventional Radiology, and the ACC/AHA Task Force on Practice Guidelines (Writing Committee to Develop Guidelines for the Management of Patients With Peripheral Arterial Disease): endorsed by the American Association of Cardiovascular and Pulmonary Rehabilitation; National Heart, Lung, and Blood Institute; Society for Vascular Nursing; TransAtlantic Inter-Society Consensus; and Vascular Disease Foundation. Circulation 2006;113:e463-654.

2. Sakalihasan N, Limet R, Defawe OD. Abdominal aortic aneurysm. Lancet 2005;365:1577-89.

3. Erbel R, Aboyans V, Boileau C, et al. 2014 ESC Guidelines on the diagnosis and treatment of aortic diseases: document covering acute and chronic aortic diseases of the thoracic and abdominal aorta of the adult. The Task Force for the Diagnosis and Treatment of Aortic Diseases of the European Society of Cardiology (ESC). Eur Heart J 2014;35:2873-926.

4. Han YS, Zhang J, Xia Q, et al. A comparative study on the medium-long term results of endovascular repair and open surgical repair in the management of ruptured abdominal aortic aneurysms. Chin Med J (Engl) 2013;126:4771-9.

5. Yin $M$, Zhang J, Wang $Y$, et al. Deficient CD4+CD25+ $T$ regulatory cell function in patients with abdominal aortic aneurysms. Arterioscler Thromb Vasc Biol 2010;30:1825-31.

6. Davis FM, Rateri DL, Daugherty A. Abdominal aortic aneurysm: novel mechanisms and therapies. Curr Opin Cardiol 2015;30:566-73.

7. Li J, Krishna SM, Golledge J. The potential role of kallistatin in the development of abdominal aortic aneurysm. Int J Mol Sci 2016. doi: 10.3390/ijms17081312.

8. Han Y, Tanios F, Reeps C, et al. Histone acetylation and histone acetyltransferases show significant alterations in human abdominal aortic aneurysm. Clin Epigenetics 
2016;8:3.

9. Yao QJ, Sang L, Lin M, et al. Mettl3-Mettl14 methyltransferase complex regulates the quiescence of adult hematopoietic stem cells. Cell Res 2018;28:952-4.

10. Wang X, Lu Z, Gomez A, et al. N6-methyladenosinedependent regulation of messenger RNA stability. Nature 2014;505:117-20.

11. Li Z, Weng H, Su R, et al. FTO plays an oncogenic role in acute myeloid leukemia as a N6-methyladenosine RNA demethylase. Cancer Cell 2017;31:127-41.

12. Gilbert WV, Bell TA, Schaening C. Messenger RNA modifications: form, distribution, and function. Science 2016;352:1408-12.

13. Roignant JY, Soller M. m6A in mRNA: an ancient mechanism for fine-tuning gene expression. Trends Genet 2017;33:380-90.

14. Pan Y, Ma P, Liu Y, et al. Multiple functions of m6A RNA methylation in cancer. J Hematol Oncol 2018;11:48.

15. Zhang S, Zhao BS, Zhou A, et al. m6A demethylase ALKBH5 maintains tumorigenicity of glioblastoma stem-like cells by sustaining FOXM1 expression and cell proliferation program. Cancer Cell 2017;31:591-606.e6.

16. Ma JZ, Yang F, Zhou CC, et al. METTL14 suppresses the metastatic potential of hepatocellular carcinoma by modulating N6-methyladenosine-dependent primary microRNA processing. Hepatology 2017;65:529-43.

17. Du K, Zhang L, Lee T, et al. m6A RNA methylation controls neural development and is involved in human diseases. Mol Neurobiol 2019;56:1596-606.

18. Wu Y, Xie L, Wang M, et al. Mettl3-mediated m6A RNA methylation regulates the fate of bone marrow mesenchymal stem cells and osteoporosis. Nat Commun 2018;9:4772.

19. Meyer KD, Saletore Y, Zumbo P, et al. Comprehensive analysis of mRNA methylation reveals enrichment in 3' UTRs and near stop codons. Cell 2012;149:1635-46.

20. Panneerdoss S, Eedunuri VK, Yadav P, et al. Cross-talk among writers, readers, and erasers of $\mathrm{m} 6 \mathrm{~A}$ regulates cancer growth and progression. Sci Adv 2018;4:eaar8263.

21. Liu ZX, Li LM, Sun HL, et al. Link between m6A modification and cancers. Front Bioeng Biotechnol 2018;6:89.

22. Meng TG, Lu X, Guo L, et al. Mettl14 is required for mouse postimplantation development by facilitating epiblast maturation. FASEB J 2019;33:1179-87.

23. Koranda JL, Dore L, Shi H, et al. Mettl14 is essential for epitranscriptomic regulation of striatal function and learning. Neuron 2018;99:283-92.e5.
24. Feng Z, Li Q, Meng R, et al. METTL3 regulates alternative splicing of MyD88 upon the lipopolysaccharideinduced inflammatory response in human dental pulp cells. J Cell Mol Med 2018;22:2558-68.

25. Zhang Y, Guo F, Zhao R. Hepatic expression of FTO and fatty acid metabolic genes changes in response to lipopolysaccharide with alterations in m6A modification of relevant mRNAs in the chicken. Br Poult Sci 2016;57:628-35.

26. Tang D, Han Y, Lun Y, et al. Y chromosome loss is associated with age-related male patients with abdominal aortic aneurysms. Clin Interv Aging. 2019;14:1227-41.

27. Chaikof EL, Dalman RL, Eskandari MK, et al. The Society for Vascular Surgery practice guidelines on the care of patients with an abdominal aortic aneurysm. J Vasc Surg 2018;67:2-77.e2.

28. Tong J, Flavell RA, Li HB. RNA m6A modification and its function in diseases. Front Med 2018;12:481-9.

29. Mathiyalagan P, Adamiak M, Mayourian J, et al. FTOdependent N6-methyladenosine regulates cardiac function during remodeling and repair. Circulation 2019;139:518-32.

30. Chen J, Sun Y, Xu X, et al. YTH domain family 2 orchestrates epithelial-mesenchymal transition/ proliferation dichotomy in pancreatic cancer cells. Cell Cycle 2017;16:2259-71.

31. Shi H, Wang X, Lu Z, et al. YTHDF3 facilitates translation and decay of N6-methyladenosine-modified RNA. Cell Res 2017;27:315-28.

32. Nishizawa Y, Konno M, Asai A, et al. Oncogene $\mathrm{c}-\mathrm{Myc}$ promotes epitranscriptome $\mathrm{m} 6 \mathrm{~A}$ reader YTHDF1 expression in colorectal cancer. Oncotarget 2018;9:7476-86.

33. Dominissini D, Moshitch-Moshkovitz S, Schwartz S, et al. Topology of the human and mouse m6A RNA methylomes revealed by m6A-seq. Nature 2012;485:201-6.

34. Mellak S, Ait-Oufella H, Esposito B, et al. Angiotensin II mobilizes spleen monocytes to promote the development of abdominal aortic aneurysm in Apoe-/- mice. Arterioscler Thromb Vasc Biol 2015;35:378-88.

35. Raffort J, Lareyre F, Clément M, et al. Monocytes and macrophages in abdominal aortic aneurysm. Nat Rev Cardiol 2017;14:457-71.

36. Bonnard T, Serfaty JM, Journé C, et al. Leukocyte mimetic polysaccharide microparticles tracked in vivo on activated endothelium and in abdominal aortic aneurysm. Acta Biomater 2014;10:3535-45.

37. Li HB, Tong J, Zhu S, et al. m6A mRNA methylation 
controls T cell homeostasis by targeting the IL-7/STAT5/ SOCS pathways. Nature 2017;548:338-42.

38. Poissonnier L, Villain G, Soncin F, et al. miR126$5 \mathrm{p}$ repression of ALCAM and SetD5 in endothelial cells regulates leucocyte adhesion and transmigration.

Cite this article as: He Y, Xing J, Wang S, Xin S, Han Y, Zhang J. Increased m6A methylation level is associated with the progression of human abdominal aortic aneurysm. Ann Transl Med 2019;7(24):797. doi: 10.21037/atm.2019.12.65
Cardiovasc Res 2014;102:436-47.

39. Reeps C, Pelisek J, Seidl S, et al. Inflammatory infiltrates and neovessels are relevant sources of MMPs in abdominal aortic aneurysm wall. Pathobiology 2009;76:243-52. 


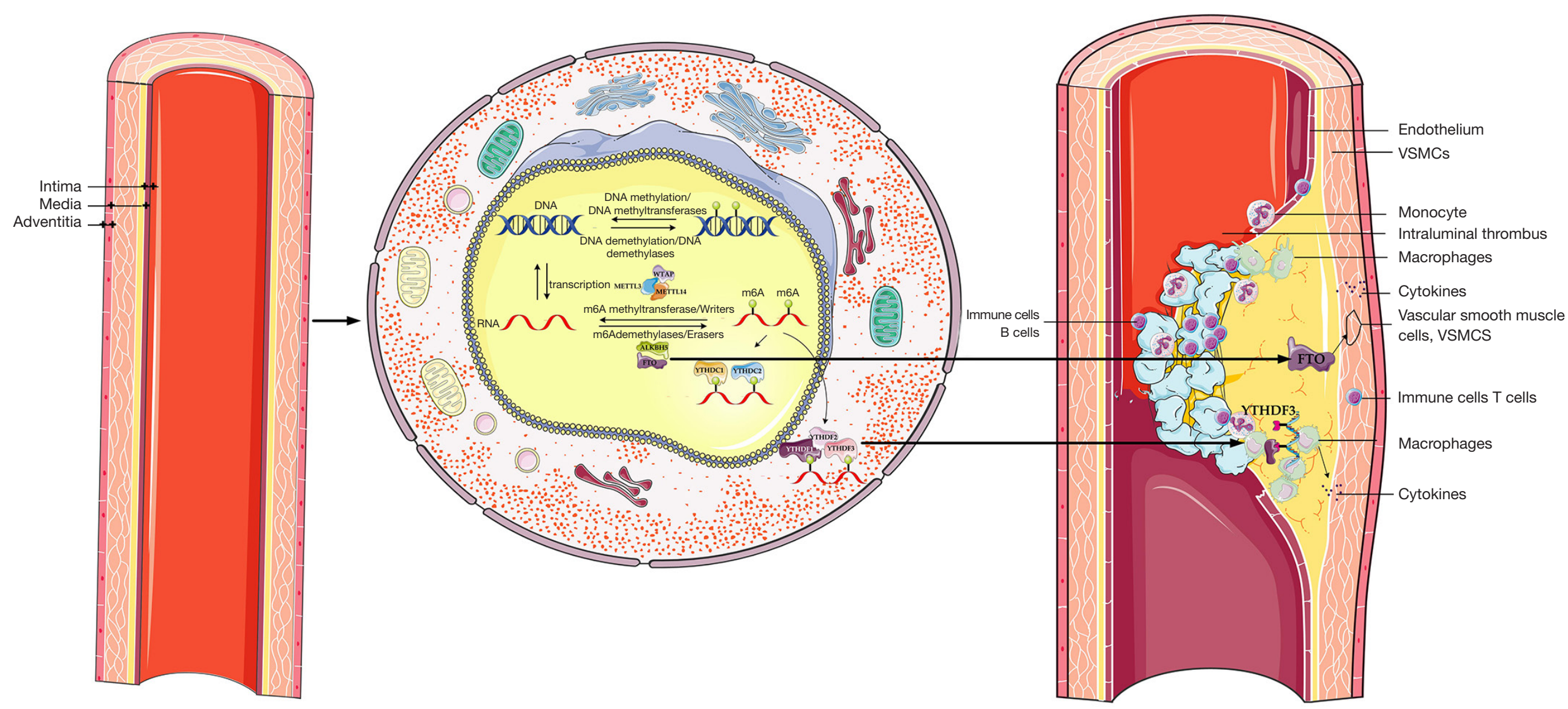

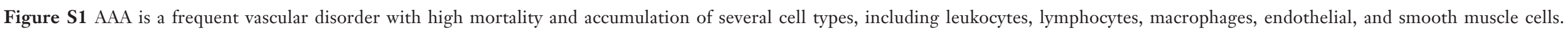

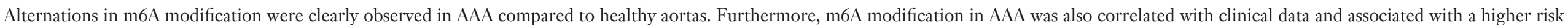
of AAA rupture. m6A, N6-methyladenosine; AAA, abdominal aortic aneurysm; VSMC, vascular smooth muscle cell. 